\title{
APLICACIÓN DEL MODELO DE BLACK - LITTERMAN A LA SELECCIÓN DE PORTAFOLIOS INTERNACIONALES
}

\author{
APPLICATION MODEL BLACK - LITTERMAN \\ A SELECTION OF INTERNATIONAL PORTFOLIOS \\ Rafael Cruz Salazar* \\ Arcadio Clement P.** \\ Universidad Nacional Autónoma de México-UNAM / Distrito Federal - México \\ [Recepción: Marzo de 2014/ Conformidad: Mayo 2014]
}

\section{RESUMEN}

Se muestra como el modelo de Black - Litterman en la construcción de un portafolio con activos internacionales ayuda a mejorar la aplicación de los principios en que se basa la teoría de selección de portafolios propuesta por Markowitz, permitiendo un enfoque más robusto para obtener portafolios diversificados cuyas ponderaciones son modificadas de acuerdo con las expectativas que se tengan sobre el desempeño de cada uno de los activos. El principal beneficio del modelo Black - Litterman es que el administrador del portafolio puede hacer el análisis iterativamente hasta obtener un portafolio coherente con sus expectativas, lo que representa una excelente alternativa para aquellos inversionistas que buscan analizar cada uno de los elementos que pueden influir en el desempeño de sus portafolios.

Palabras clave:

Black - Litterman; Markowitz; riesgo; administración de portafolios.

\begin{abstract}
It shows how the model Black - Litterman in building a portfolio of international assets helps improve the application of the principles on which the theory of portfolio selection proposed by Markowitz is based, allowing a more robust approach for diversified portfolios whose weights are modified according to the expectations that have on the performance of each of the assets. The main benefit of the model Black Litterman is that the portfolio manager can do the analysis iteratively until a portfolio consistent with their expectations, which is an excellent alternative for those investors looking to analyze each of the elements that can influence the performance of their portfolios.
\end{abstract}

\section{Keywords:}

Black - Litterman; Markowitz; risk; portfolio management.

\footnotetext{
* Maestro en Finanzas con especialidad en Finanzas Bursátiles, UNAM. Email:rafael.cruz.salazar@gmail.com

** Master of Business Administration, Nova Southeastern University. Licenciado en Banca y Finanzas, Licenciatura en Administración de Empresas, Universidad Latinoamericana de Ciencia y Tecnología. Email: clement1958@gmail.com
} 


\section{INTRODUCCIÓN}

Existen diversas teorías y modelos de administración de portafolios, todos ellos desarrollados a lo largo del tiempo, y es evidente que tanto la selección como la administración de dichos portafolios no se deben de llevar de una manera banal y superficial, sino que, por el contrario, requiere de un alto grado de estudio, técnica e información. Desde que se comenzaron a formular los diferentes métodos de administración de portafolios, siempre un primer elemento a considerar es el cálculo del famoso binomio rentabilidad - riesgo, y esto nos exige tener información y conocer aspectos de la economía, del sector en el que se va a invertir, así como también de la empresa o del título en el que vamos a invertir.

La diversificación de los portafolios de inversión y su optimización es un tema central en el ambiente financiero, los primeros modelos de la teoría de portafolios se formularon en un ambiente nacional. Sin embargo, actualmente es fácil para un inversionista acceder a títulos de valores de diversos mercados financieros alrededor del mundo, lo cual ha llevado al desarrollo de nuevos modelos que incorporen tanto los factores nacionales como internacionales.

$\mathrm{Y}$ es que los riesgos a los que se enfrentan los inversionistas en el ámbito internacional son un tanto más complejos que en el ámbito nacional, de esta manera el comportamiento de un activo en un mercado local es diferente al que tendría en un mercado extranjero, puesto que existen factores cuantitativos y cualitativos que no siempre tienen el mismo impacto en un mercado que en otro, de entre estos factores podemos destacar uno muy común a todos ellos, el cual es el riesgo cambiario, quizás este represente uno de los principales riesgos que existen y que los inversionistas toman mucho en cuanto al momento de invertir en un mercado extranjero.

Por ello, el objetivo de esta investigación es analizar la manera en que el modelo de Black - Litterman administra los riesgos en los portafolios internacionales. Determinando el impacto que tiene la volatilidad del tipo de cambio en la formación, administración y rendimiento de un portafolio de inversión internacional. Y desarrollar y proponer un modelo de administración tanto activa como pasiva de los portafolios internacionales, para que el inversionista pueda llevar a cabo la aplicación de esta investigación en la formación de portafolios de inversión, de una manera sistemática y analítica, y así poder obtener mayores beneficios a su inversión.

\section{TEORÍAS DE FORMACIÓN DE PORTAFOLIOS}

Desde su aparición el modelo de Markowitz ha tenido una gran aceptación a nivel teórico, ya que sentó las bases de la Teoría Moderna de Portafolios, es aquí donde se asocia el riesgo con el rendimiento y cómo un conjunto de combinaciones de esta dualidad genera portafolios eficientes. Sin embargo, muchos de los supuestos que se planteaban dentro de este modelo en la práctica presentaban algunas limitantes tales como, la racionalidad de los inversionistas, entre otros.

El modelo de Asignación de Precios de Capital (Capital Asset Pricing Model CAPM), hace un gran aporte al explicar el comportamiento de una acción en función del comportamiento del mercado, y surge un concepto importante: el factor beta $b$ el cual mide la covarianza del rendimiento de la acción respecto del índice del mercado. Sin embargo, la beta no explica en sí misma, el comportamiento que ha detener la acción respecto del mercado.

La teoría de Precios de Arbitraje (Arbitrage pricing theory APT) es una alternativa al Modelo de Asignación de Precios de Capital (CAPM). El APT permite múltiples factores de riesgo y no requiere la identificación del portafolio del mercado. En su forma más general el APT provee una relación aproximada entre el rendimiento esperado de un activo con $n$ número de factores no identificados.

A pesar de las limitaciones que podrían presentar los modelos de construcción de portafolios, estos han tenido gran aceptación, que incluso se desarrollaron modelos para el entorno internacional, de tal forma, que surgieron los modelos ICAPM o IAPT con los cuales se incorporaron los factores internacionales para así poder llevar a cabo la construcción de portafolios internacionales. Sin embargo, al igual que sucedía con su contraparte nacional, estos modelos seguían teniendo sus limitantes internacionales de- 
rivadas de las limitantes postuladas en los entornos nacionales.

\section{EL MODELO DE BLACK - LITTERMAN}

El modelo de Black - Litterman parte del modelo de Markowitz, y está basado en modelos bayesianos, la importancia de esto radica básicamente en la posibilidad de incorporar conocimiento extra muestral "a priori" en la estimación de los modelos.

En el caso de la estimación de los rendimientos esperados a partir de la información muestral se tiene que, según el teorema de Bayes:

$$
P(A \mid B)=\frac{P(B \mid A) P(A)}{P(B)}
$$

$P(A \mid B)$ : Es la probabilidad condicional de A dado B, conocida también como la condición a posteriori.

$P(B \mid A)$ : Es la probabilidad condicional de B dado A.

$P(A)$ : La probabilidad de $\mathrm{A}$, también conocida como probabilidad a priori.

$P(B)$ : La probabilidad de B también llamada constante normal.
La importancia del modelo de Black - Litterman radica en la incorporación de elementos subjetivos e intuitivos, como son las expectativas que tiene el inversionista acerca del rendimiento esperado de un activo. el modelo también tiene como base los modelos CAPM y APT como combinación a priori, aunque el CAPM necesariamente no refleja de forma exacta las expectativas del mercado, constituye un punto de referencia para comparar las expectativas propias y determinar qué oportunidades de inversión existen.

El modelo le da la posibilidad al inversionista de asumir un riesgo alto o bajo para un activo determinado, dependiendo de la confianza que tenga sobre la expectativa correspondiente a ese activo. También diferencia entre la magnitud de la expectativa y el grado de certeza que se tiene sobre la misma.

Aplicando el teorema de Bayes al problema de la fusión de las distribuciones previas y condicionales para crear una nueva distribución posterior de los rendimientos de los activos y dadas las siguientes ecuaciones de probabilidad:

$$
\begin{gathered}
P\left((A \mid B) \sim \mathbb{N}\left(\left[\Omega^{-1} \mu+n S^{-1} x\right]^{\tau}\left[\Omega^{-1}+n S^{-1}\right]^{-1},\left(\Omega^{-1}+n S^{-1}\right)^{-1}\right)\right. \\
P(A) \sim \mathbb{N}(\Pi, \tau S) \\
P(B \mid A) \sim \mathbb{N}\left(P^{-1} Q,\left[P^{T} \Omega^{-1} P\right]^{-1}\right)
\end{gathered}
$$

Donde:

$\mathrm{S}$ es la varianza de la media sobre $\mathrm{n}$ número de muestras.

$\mathrm{P}$ es la matriz que selecciona los activos que hacen parte de una opinión.

$\mathrm{Q}$ una matriz de $\mathrm{k} \times 1$ de los rendimientos para cada punto de vista. $Q$ es el vector de opiniones, es decir que contiene el retorno esperado para cada portafolio. $\Omega$ es una matriz kx k de la varianza de los puntos de vista.

$\tau$ es el parámetro escalar que se puede interpretar cómo el grado de incertidumbre del inversionista sobre la validez del CAPM.

$\mu$ como una variable aleatoria que se distribuye de la siguiente manera:

$$
\mu \sim \mathbb{N}\left(\pi, \Sigma_{\pi}\right)
$$

$\pi$ es nuestra estimación de la media.

$\Sigma_{\pi}$ es nuestra varianza de la estimación de la media de los rendimientos.

Podemos aplicar el teorema de Bayes y derivar nuestra fórmula para la posterior distribución del rendimiento de los activos, de tal manera que obtenemos:

$$
P\left((A \mid B) \sim \mathbb{N}\left(\left[\tau \Sigma^{-1} \Pi+P^{\mathrm{T}} \Omega^{-1} Q\right]\left[(\tau \Sigma)^{-1}+P^{T} \Omega^{-1} P\right]^{-1},\left((\tau \Sigma)^{-1} P^{T} \Omega^{-1} P\right)^{-1}\right)\right.
$$

Despejando para $\Pi$ :

$$
\Pi=\delta \Sigma w
$$

A fin de utilizar la fórmula anterior tenemos que tener un valor para $\delta$, el coeficiente de aversión de riesgo del mercado. Black - Litterman utilizan el índice de Sharpe cercano a 0.5. 


$$
\delta=\left(\mathrm{E}(\mathrm{r})-\mathrm{r}_{\mathrm{f}}\right) / \sigma^{2}
$$

$\mathrm{E}(\mathrm{r})=$ Rendimiento total del portafolio de mercado.

$\mathrm{r}_{\mathrm{f}}=$ Tasa libre de riesgo.

$\sigma^{2}=$ Varianza del portafolio.

Como se puede observar, la información histórica no influye directamente en la determinación de $\Pi$. La variable $\delta$ se puede interpretar como el parámetro de aversión al riesgo.

\section{PORTAFOLIOS INTERNACIONALES}

Una vez estudiada la metodología del modelo Black - Litterman, se aplicó a los diferentes mercados financieros para así poder obtener los portafolios óptimos y proceder a desarrollar el modelo para el mercado accionario internacional.

Nos situaremos en el papel de un inversionista mexicano, que pretende realizar una inversión en diferentes países, en específico: Alemania, Brasil, España, Inglaterra y Estados Unidos. El periodo comprendido de estudio para la formación de portafolios es de 2000 a 2009.

El nivel de aversión al riesgo en el modelo de Black - Litterman determina en gran medida la formación del portafolio de inversión, esta parte subjetiva dentro de dicho modelo enriquece en gran medida la teoría de portafolios de inversión, puesto que con Markowitz no se considera esta aversión del inversionista, sino hasta el momento de tener estructurado la frontera eficiente de inversión, el inversionista decidía que portafolio tomaría, en cambio en el modelo de Black -Litterman, el inversionista ya conoce su nivel de aversión al riesgo y lo utiliza como factor para calcular el único portafolio óptimo.

Retomando la variable $\delta$, como el coeficiente de aversión de riesgo del mercado en el modelo de Black - Litterman, se tomaron los portafolios bases obtenidos mediante Markowitz (periodo 2000-2009), y se calcularon los portafolios mediante la metodología Black - Litterman con diferentes niveles de aversión al riesgo para posteriormente evaluar su rendimiento y por consiguiente emitir si realmente representa una mejor metodología al momento de llevarla a cabo en el ámbito internacional. Los niveles de aversión al riesgo que se consideraron son los siguientes:

\section{Tabla $\mathbf{N}^{\circ} 1$}

\section{Niveles de aversión al riesgo}

\begin{tabular}{|c|}
\hline Nivel de aversión \\
\hline 0.25 \\
\hline 0.5 \\
\hline 1.5 \\
\hline 1.75 \\
\hline
\end{tabular}

Los rendimientos en los portafolios alemanes no presentan un cambio marginal en los niveles de 1.50 a 1.75 , por lo que se infiere que a un mayor nivel de aversión al riesgo dentro de este mercado conlleva a una estabilización en el rendimiento del portafolio. Sin embargo entre menor sea el nivel de aversión al riesgo mayor sera el rendimiento esperado, esto se debe a que se concentra la mayor parte hacia una acción la cual se espera que de mayores rendimientos y sea más riesgosa, lo cual comprueba una vez más la relación riesgo- rendimiento.

\section{Gráfico $\mathrm{N}^{\circ} 1$}

Rendimientos Portafolio de Alemania

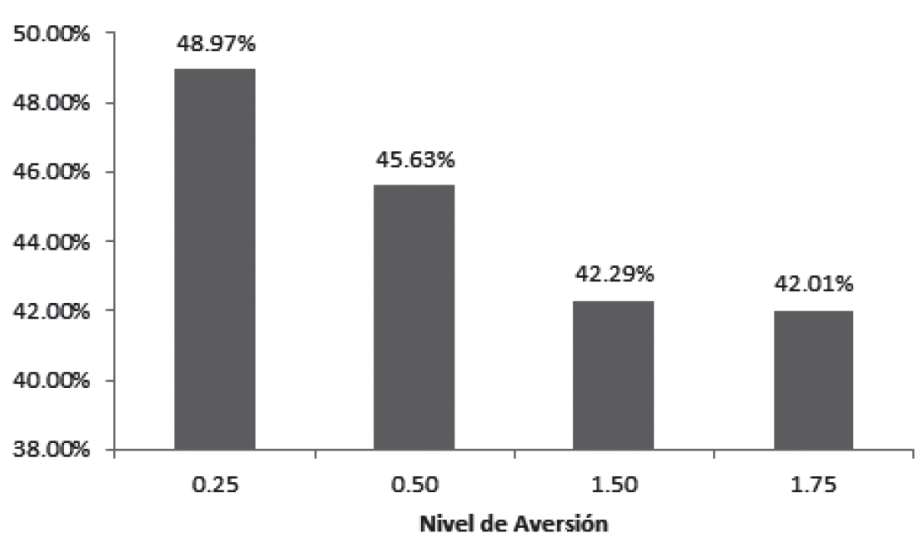

Fuente: Reuters, enero 2009 - abril 2011. Elaboración: Propia. 
En resumen, un inversionista poco adverso al riesgo obtendrá una diferencia de cinco puntos porcentales de un inversionista adverso al riesgo, este diferencial de rendimiento supera al obtenido incluso mediante la divisa, de ahí la importancia de conocer el nivel de aversion de cada inversionista.

Brasil presenta el mismo caso solo que el cambio marginal más notorio se da al pasar de un nivel de 1.50 a 1.75; es decir, el inversionista que decida invertir en el mercado brasileño y que sea sumamente adverso al riesgo, obtendrá un rendimiento sumamente diferente de un inversionista poco adverso al riesgo, también hay que recordar que Brasil es uno de los mercados emergentes de suma importancia con lo cual hace que más notoria estas diferencias, pues las variaciones que sufre tanto su moneda como sus acciones son drásticas a diferencia de otras economías desarrolladas.

En otras palabras, un inversionista adverso al riesgo obtiene un diferencial de dos puntos porcentuales menos que uno poco adverso, lo cual representa un cambio menos significativo si lo comparamos con el obtenido por la divisa.

\section{Gráfico $\mathrm{N}^{\circ} 2$}

Rendimientos Portafolio de Brasil

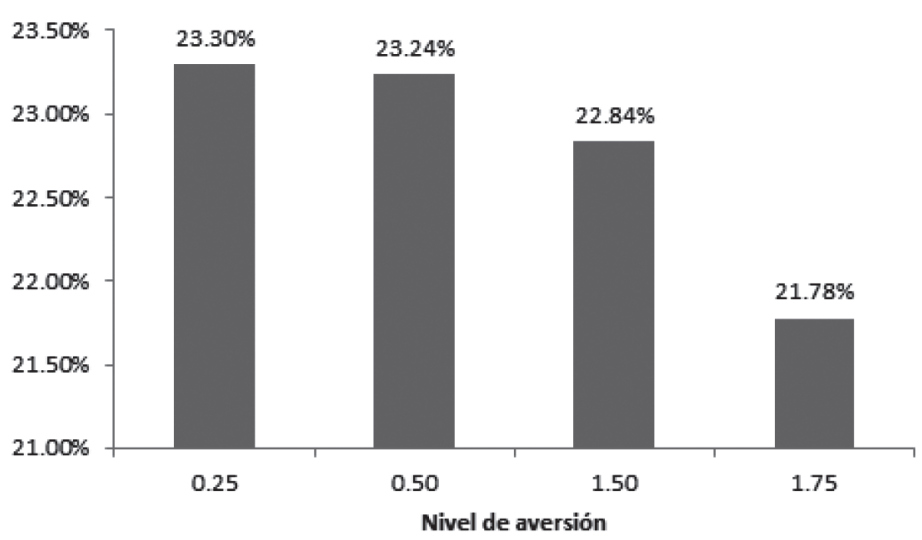

Fuente: Reuters, enero 2009 - abril 2011. Elaboración: Propia.

El mercado español es un mercado que presenta rendimientos negativos producto ya analizado de las afectaciones de la crisis financiera. Sin embargo, si nos fijamos detenidamente en las pérdidas, se observa un fenómeno sumamente interesante, a mayor aversión al riesgo mayores son las pérdidas, es decir, un inversionista amante del riesgo hubiese obtenido resultados menos peores que uno que no lo era. Aunque el cambio marginal es de un punto porcentual, lo interesante del caso se centra en el hecho de que el portafolio les confiere mayores beneficios a los amantes del riesgo, es decir la teoría riesgo-rendimiento prevalece incluso en los mercados con rendimientos negativos. 


\section{Gráfico $\mathrm{N}^{\circ} 3$ \\ Rendimiento Portafolio de España}

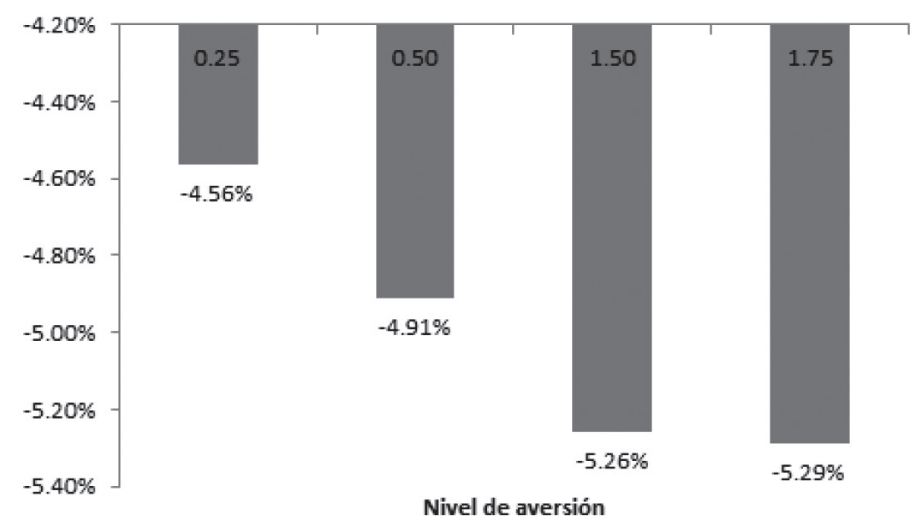

Fuente: Reuters, enero 2009 - abril 2011. Elaboración: Propia.

De igual forma, el mercado inglés es un mercado que otorga mayores beneficios a los amantes del riesgo, solo que al igual que España, el cambio marginal es mínimo, de tan solo un punto porcentual, producto de una similitud en las varianzas de las acciones que conforman dicho portafolio, así como también, de una estabilidad en el mercado inglés, que si es bien afectado por la crisis económica, los estragos fueron posteriormente neutralizados debido a su fortaleza financiera.

\section{Gráfico $\mathrm{N}^{\circ} 4$}

\section{Rendimientos Portafolio de Inglaterra}

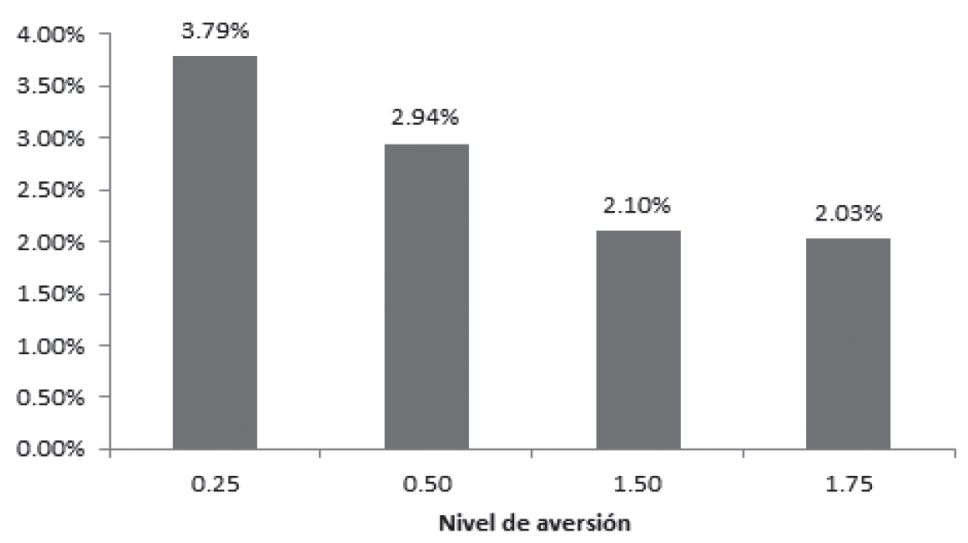

Fuente: Reuters, enero 2009 - abril 2011. Elaboración: Propia.

Por último en el mercado estadounidense, siguiendo la misma tendencia y obedeciendo a los establecido por la teoría, presenta mayores beneficios a los inversionistas amantes del riesgo, solo que al igual que el mercado inglés también su cambio marginal no es ni siquiera de medio punto porcentual, lo cual nos habla de varianzas muy similares entre las acciones que conforman dicho portafolio.
Finalmente, podemos mencionar que la importancia del modelo de Black - Litterman se centra en la subjetividad de las opiniones y en el dinamismo que presenta al momento no solo de conformar los portafolios sino de evaluar sus rendimientos y sobre todo los aspectos que podrían afectar dicho rendimientos, de tal forma que podamos modificarlo de acuerdo a las necesidades y características de cada inversionista. 


\section{Gráfico $\mathrm{N}^{\circ} 5$ \\ Rendimientos Portafolio de Inglaterra}

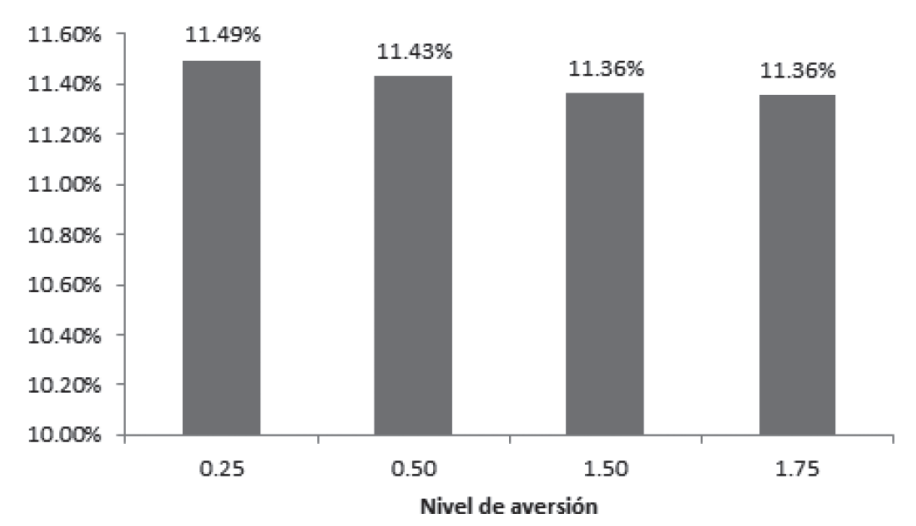

Fuente: Reuters, enero 2009 - abril 2011. Elaboración: Propia.

\section{CONCLUSIONES}

1. Se ha concluido que la aplicación del Modelo de Black - Litterman, le permite de una mejor forma al inversionista, identificar los elementos que afectan su portafolio de inversión dentro de los mercados globales. Cumpliendo así el objetivo planteado, es decir, analizar la manera en que el Modelo de Black - Litterman administra los riesgos en los portafolios internacionales, mediante la determinación del impacto que tiene la volatilidad del tipo de cambio en la formación, administración y rendimiento de un portafolio de inversión internacional.

2. El modelo Black - Litterman es un modelo de construcción de portafolios muy útil, particularmente, para aquellos que al momento de formar un portafolio tienen alguna estrategia de inversión. Un aspecto importante al que se llegó es que el mayor beneficio del modelo está en enfrentar los problemas tradicionales de las decisiones de inversión de una manera sistemática y transparente.

3. El beneficio del modelo Black - Litterman Internacional, es que el administrador del portafolio lo pueda usar de forma iterativa, hasta que obtenga resultados coherentes con sus expectativas. Las expectativas llevadas a cabo en este estudio, no necesariamente reflejan las expectativas de un inversionista en específico, puesto que solo reflejan las opiniones de un individuo, sin embargo, esto no significa que por ende sean incorrectas, ya que esto es lo que enriquece al modelo de Black - Litterman.

4. El hecho de que cada individuo perciba el comportamiento del mercado de una forma diferente a otro inversionista nos habla de una diversidad de opiniones infinitas. Sin embargo, hay que recordar que en la mayoría de los casos estas expectativas son muy similares. Como se pudo observar en este estudio, otra de las conclusiones a las que se llegó es que las expectativas también se pueden modelar, con esto se lograría un mayor alcance al momento de eliminar cualquier elemento subjetivo del modelo Black - Litterman.

5. En resumen, el modelo Black - Litterman aplicado a la selección de portafolios internacionales representa una excelente alternativa para aquellos inversionistas que buscan analizar cada uno de los elementos que influyen en el desarrollo de sus portafolios de inversión, así como también, debido a esta aplicación surgen diversas líneas de investigación, las cuales ya fueron presentadas, y que conlleva a una serie de estudios del impacto de dichas variables en los estudios de los mercados financieros. 


\section{REFERENCIAS BIBLIOGRÁFICAS}

1. Black, F. and Litterman, R. (1991). Global Asset Allocation with Equities, Bonds, and Currencies. Fixed Income Research, Goldman Sachs.

2. Black, F. and Litterman, R. (1992). Global Portfolio Optimization. Financial Analysts Journal, September-October, 28-43.
3. He, G. and Litterman, R. (1999). The Intuition Behind Black-Litterman Model Portfolios. Goldman Sachs Quantitative Resources Group.

4. Litterman, R. (ed.) (2003). Modern Investment Management: An Equilibrium Approach. John Wiley, New Jersey. 